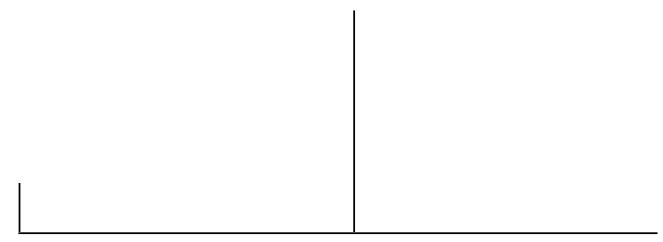

Rev. Latinoam. Psicopat. Fund., São Paulo, v. 15, n. 4, p. 766-780, dezembro 2012

\title{
De l'insensé du geste suicidaire à la condensation signifiante de la scène suicidaire
}

Joanne André

Bernard Chouvier

Le geste suicidaire est une énigme, "comment le moi peut consentir à son auto-destruction" s'interroge Freud en 1915. Partant de ce constat théorique et clinique, cet article, en s'appuyant sur l'histoire de Chantal, propose une voie de réflexion sous forme d'après-coup. Le geste suicidaire inclut une scène qui constitue une première répétition ou encore le second temps du trauma. Cette réactualisation traumatique peut être suivie d'un mouvement d'historisation de l'insensé originaire.

Mots clés: Geste suicidaire, paradoxe, après-coup, scène suicidaire 
Ma contribution à l'énigme suicidaire s'appuie sur des rencontres au lendemain d'un geste suicidaire. Ces rencontres ont lieu aux urgences, les sujets sont essentiellement des femmes dont le fonctionnement psychique est ordinairement névrotique, et dont l'état dépressif n'est pas au premier plan, même s'il est plus ou moins présent.

Au réveil les mots énoncés par les patients: “je n’en pouvais plus", "j'étais à bout"; et aussi: "je ne sais plus, je ne sais pas", ne cherchent pas encore le sens, ils en expriment le manque, l'absence. Manque, absence de mots pour dire le sentiment vécu, dont la trace est parfois inaccessible au souvenir, quand l'expérience est en mal d'inscription dans le texte et dans la tête, dans la langue maternelle et dans Psyché.

Le paradoxe, l'étrangeté, envahit la situation: la rencontre a lieu car l'un a désiré ne pas, ne plus être. De l'urgence somatique à l'intervention "psy" aux urgences, il y a un nécessaire changement de rythme, de temps; il s'agit de donner du temps à une situation qui par définition n'en disposait pas.

Paradoxes et effets d'étrangeté quand une femme, au réveil d'un geste suicidaire, dit son "calvaire" depuis plusieurs mois, calvaire dont elle "voit le bout" au travers de son projet de changement pour "vivre sa vie": que signifie son geste suicidaire entre fin du calvaire et début de "sa vie"? Quatre jours plus tard, elle vient au rendez-vous proposé, elle est méconnaissable physiquement reconstruite, psychiquement debout, elle semble avoir trouvé un nouvel élan... Comment comprendre? 


\section{"Vivre"}

Chantal a une quarantaine d'année, elle est mariée et a deux enfants de cette union, un garçon de 10 ans et une fille de trois ans sa cadette. Arrivée aux urgences la veille, en fin de soirée, elle est hospitalisée pour une tentative de suicide ou "ingestion médicamenteuse volontaire" (IMV), dite grave par les urgentistes. Il s'agit d'un premier geste suicidaire chez cette femme. J'ai rencontré Chantal à deux reprises pendant le temps de son hospitalisation qui a duré 48 heures. Elle viendra ensuite aux entretiens que je lui ai proposés. Ce qui suit retrace son histoire au long des entretiens, selon la chronologie, non des faits, mais de son discours.

Chantal pleure beaucoup, se tient la tête, se recroqueville et se raconte sans discontinuer. Au fil des premiers entretiens, elle semble se reconstruire physiquement et psychiquement, et ceci très rapidement, presque soudainement.

Elle vit un calvaire avec son mari qui la harcèle, dit-elle. Elle se sent épiée au travail, à la maison, elle ne le supporte plus et "elle ne se supporte plus face à lui", elle a décidé de le quitter. La séparation, décision qu'elle a prise il y a quelques mois, va avoir lieu dans quelques jours, elle déménage avec ses enfants car lui ne veut pas partir. Pour elle se profile "la fin de son calvaire".

"J'étais à bout... au bord du gouffre", dit-elle en évoquant le moment de son geste. La scène est au départ récurrente: son mari fait intrusion dans son intimité en pénétrant dans le lieu où elle s'est isolée. Il lui fait des reproches, lui pose des questions, le ton monte entre les époux et, cette fois, elle avale l'ensemble des médicaments qui lui avait été prescrit deux années plutôt, suite au décès de son père; "Là, je n'en pouvais plus", ajoute-t-elle en larmes.

Le père de Chantal s'est suicidé. C'était, dit-elle, un homme de longue date dépressif dont elle était très proche. Régulièrement, il passait chez elle, venant de chez lui à pied. Ils parlaient longuement et il rentrait chez lui. Le jour de son suicide, le père de Chantal est passé chez elle mais il n'est pas rentré chez lui, "il a poursuivi sa route et il s'est suicidé". Chantal se reproche de ne pas avoir su, vu et de l'avoir laissé repartir. Elle dit, "je n'ai pas pris le temps", “je n'étais pas là" (présente physiquement, c'est d'une absence psychique dont il est question), et l'on entend: "par ma faute, il s'est tué, je l'ai tué". À plusieurs reprises, m'interrogeant du regard, elle répètera ce qu'elle "a compris depuis: quand quelqu'un veut vraiment mourir, on ne peut pas l'en empêcher". Cet énoncé, cette tentative de déculpabilisation, restait, à l'entendre, une bouée incertaine. Puis, elle évoque sa mère tyrannique et responsable, à ses yeux, de la souffrance de son père. Suite au décès de celui-ci, Chantal a décidé de quitter son mari pour "vivre sa vie". Elle se souvient: "avant notre mariage, j' ai voulu le quitter, je le lui ai dit, il m'a répondu qu'il se suiciderait... je me suis mariée". 


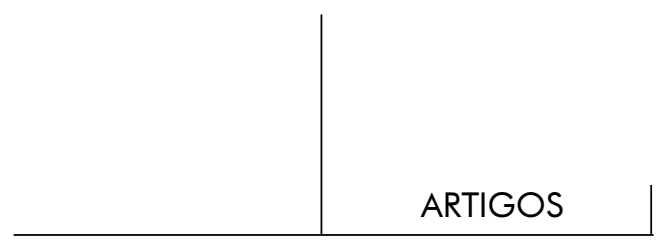

Je revois cette femme après la fin de son hospitalisation. Nous nous rencontrons dans un cadre différent, quoique toujours à l'hôpital. Quelques jours seulement séparent les entretiens et pourtant je ne la reconnais pas. Elle est souriante, élégante et évoque quelqu'un d'assuré. L'effet d'étrangeté est saisissant. Une fois passée la surprise, notre entretien reprend aisément son fil ; c'est donc bien elle. Ses projets se sont réalisés, elle a un "chez elle", petit mais suffisant pour l'instant, un lieu où elle se sent en sécurité et à partir duquel un avenir est envisageable.

Ce récit de cas laisse voir la richesse du jeu identificatoire et sa complexité. Le geste suicidaire de Chantal s'inscrit dans une histoire où le suicide, sa menace et la séparation se confondent, de tout temps semble-t-il, au travers de la pathologie dépressive du père et de la "tyrannie" de la mère. Dans ce jeu relationnel, Chantal a occupé une place et joué un rôle particuliers auprès de son père qui se sont répétés auprès de son époux. Les relations conjugales, d'une génération à l'autre, semblent également s'inscrire dans une redite de la tyrannie, inversée peut-être, mais pas seulement car Chantal précise: "je ne me supporte plus face à lui'. Ceci interroge l'image que lui renvoie le regard de son époux: celle d'une femme tyrannique comme sa mère? Coupable de la souffrance du père, coupable de son meurtre? Toute la famille de la patiente semble être présente, condensée, au travers du geste suicidaire mais que dire du désir de mourir de Chantal?

Le geste est sérieux et pourtant, très vite, elle se récupère, tant physiquement que psychiquement. Mourir c'est retrouver son père, dit-elle, retrouver le havre de paix de cette relation à l'opposé du calvaire conjugal qui, lui, rappelle la relation à sa mère "tyrannique". Comment comprendre que cette femme attente à ses jours si près "du bout de son calvaire", au moment où elle a décidé de "vivre sa vie"? La mort que son geste appelle n'est pas celle du cadavre, la représentation en est plutôt celle d'un retour à un paradis perdu et qui, d'être perdu, ouvre sur "sa vie". Quelle collusion entre le décès du père dans la réalité et le fantasme de meurtre du père nécessaire à la constitution de chaque individu pour qu'une vie s'offre à lui?

\section{Théorie et suicide}

Tous les essais d'unification des connaissances sur le suicide, essais de théorisation, ont conclu, de nos jours encore, à l'impossibilité d'unifier ce que recouvre le terme de "suicide". La seule théorie du suicide est finalement qu'il n'y en a pas, là-dessus les auteurs s'accorderaient pour la plupart. S'il n'y a pas une théorie, il y en a plusieurs, plus que des théories ce sont des pistes de travail, des 
voies de compréhension. Peut-on aller jusqu'à la formule: "le suicide, ça n'existe pas" qui, comme la formule originaire (celle de Winnicott bien entendu), tente d'ouvrir le champ de réflexion et non de le clore, en inscrivant son objet dans une réalité autre que concrète et objectivable, une réalité psychique, humaine, relationnelle et individuelle. Il s'agirait donc de renoncer à la synthèse, à l'unité pour une pluralité comprenant des antagonismes et des points de rencontre, des paradoxes et des évidences. Renoncer à la synthèse générale afin de poursuivre la réflexion dans différents champs scientifiques.

Face à l'énigme suicidaire, aucune approche n'est à exclure, aucune à elle seule, ne peut y suffire; ce que l'on nomme "suicide" ne constitue pas un fait unifié. Ce renoncement n'est pas ancien, il ouvre sans doute d'autres voies, a priori plus parcellaires mais peut-être plus enrichissantes, invitant à repenser, à reconstruire, à l'image de l'acte suicidaire lui-même: ne pouvoir tout maîtriser, synthétiser, unifier; le sujet se sent sans issue, il s'annule.

Freud, s'il n'a pas écrit directement sur le sujet, l'a parfois rencontré, notamment à propos de la mélancolie qui reste de nos jours la référence essentielle dans ce domaine. De 1910 à 1920, Freud propose différentes voies de réflexions sur la question du suicide:

- Le suicide comme substitut à la psychose. (Les premiers psychanalystes, p. 482) Le geste suicidaire est lié à une mise en échec de la pulsion de vie dans sa fonction de liaison et de maintien de l'unité. L'issue suicidaire serait une tentative paradoxale de sauvegarder l'unité du moi. Pour sauver Psyché, le corps est dévitalisé, le clivage serait déplacé du moi à l'unité Psyché-Soma.

- Le suicide comme aboutissement de conflits psychiques (ibid., p. 491; Freud, 1920, p. 260). Le geste suicidaire serait une issue, une solution psychique, à l'image du refoulement ou encore du clivage.

- Le suicide relevant exclusivement du moi (Freud, 1915, p. 271). Une situation hors conflit; par manque de capacité conflictuelle, absence de désir, le moi se tue de n'être plus moi.

Ces voies de réflexions sur la question du suicide sont toujours actuelles: le suicide comme expression délirante, le suicide meurtre d'un autre, le suicide comme acte en lieu et place d'une parole, d'un désir.

La clinique de son côté met en exergue l'impossibilité, pour le sujet qui se réveille après un geste suicidaire, de donner, dans l'ici et maintenant, un sens à son geste. Les énoncés laissent apercevoir une ouverture psychique, un doute, une incertitude, le reste d'une béance. Les mots et les attitudes ont des effets d'étrangeté, des effets de paradoxe: l'impensable, l'impossible affleure sans se laisser saisir. 


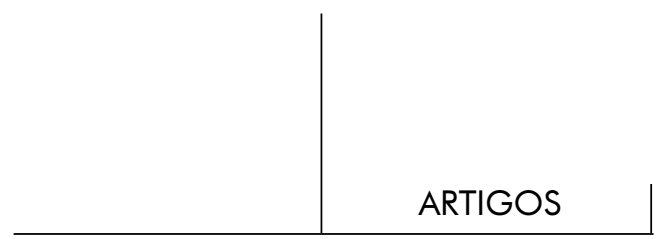

Paradoxe et crise suicidaire

L'idée du "paradoxe" n'est pas nouvelle dans la problématique suicidaire, tout au contraire c'est un point d'achoppement, à la fois prometteur et obstacle à franchir.

Deux affirmations, toutes les deux vraies, s'annulent l'une l'autre. Vouloir quitter son mari pour vivre sa vie et ne pouvoir vivre la séparation, est l'un des paradoxes de Chantal. "Mettre fin" à quelque chose en soi pour sauvegarder quelque chose de soi, en finir pour continuer, est une autre formule possible du paradoxe suicidaire. À ce paradoxe fait écho le concept de "crise suicidaire" développé en psychiatrie, plus précisément en "suicidologie".

"La crise suicidaire est une crise psychique dont le risque majeur est le suicide" (Conférence de consensus, 2000, p. 421). Cette définition, aux sonorités tautologiques, est à l'image de l'impasse vécue par le sujet en "crise"; sans issue, il tourne en rond, il répète, inlassablement. Le suicide est une issue, risquée sans nul doute, quand "l'illusion d'une identité personnelle" menace de disparaître et de laisser place à cette "béance réelle qu'est l'altérité" (McDougall, 1976, p. 140).

Le mot "crise" vient du grec "krisis" qui signifie: décision, jugement, à la croisée des chemins. Le moment d'une décision à la croisée des chemins, un moment de trouble, inconfortable voire létal, qui nécessite d'être dépassé. La crise est le passage d'un état d'équilibre à un autre. Un passage dangereux, périlleux qui engage l'avenir et remanie le passé. Un paradoxe est au coeur de toute crise: "la rupture dans la continuité" (Kaës, 1979, p. 56). La rupture est celle des limites spatiales et temporelles, entre le dedans et le dehors, entre le passé et l'actuel; la rupture est celle de la continuité même, elle ne sépare pas, elle mélange, confond, brouille, elle rend caducs les repères. Il faut inscrire cette rupture dans une continuité, tel est le paradoxe. La crise est ce travail de liaison entre avant et après, afin que le changement s'inscrive dans le temps.

La crise psychique est un état interne de tension entre les différentes instances psychiques. Des tensions dont l'évacuation est rendue impossible du fait d'un événement qui a remis en question leur mode "habituel" de traitement. Psyché lutte pour abaisser les tensions, le trop d'excitation pulsionnelle qui menace son intégrité, avec des mécanismes de défense dont l'efficacité n'est pas, n'est plus, suffisante; la déliaison menace. Pour la vie psychique, l'enjeu est celui d'un avenir potentiel. Il n'y a pas d'avenir sans passé, sans histoire, en absence de lien. Pour que s'ouvre un avenir, un projet du passé nécessite d'être abandonné, une croyance sur laquelle un avenir était bâti ne peut plus être maintenue. Ce qui donne à la crise son caractère "suicidaire" est sans doute la forme que prend ce 
renoncement, quand il constitue une perte irremplaçable au premier regard, une perte narcissique. Le seul objet, l'unique objet, non substituable, est le "moi".

La crise psychique peut être rapprochée du prototype de la situation traumatique: l'état de détresse (ou de désaide), l'Hilflosigkeit, c'est-à-dire le vécu du nouveau-né qui, du fait de son immaturité physique et psychique et en l'absence d'une réponse adaptée de son environnement, vit "un état de tension et de déplaisir intense, sans issue interne, sans fin et sans représentation" (Roussillon, 1999, p. 18). Si cet état se prolonge au-delà des capacités psychiques de liaison de l'expérience, il menace Psyché dans son organisation. Exclure de Psyché cette expérience catastrophique est une issue: un clivage, une césure, au sein de l'appareil psychique, constitue un espace hors-Psyché en son sein même, ainsi peut être traité l'intraitable en faisant mine de l'ignorer. Cette ignorance a un coût qui peut, selon les circonstances, dépasser les moyens de défense du sujet provoquant un effet de trauma. Le modèle freudien du trauma psychique est en deux temps, en deux coups, dont la chronologie est trompeuse. Effectivement, l'un et l'autre coups n'existent pas indépendamment l'un de l'autre, seule leur résonance mutuelle leur donne force et efficace, ils sont simultanés dans des temps très éloignés, dans des espaces qui s'ignorent. La crise suicidaire est un moment où le sujet est en butte avec l'effet d'un trauma, avec un afflux d'excitation pulsionnelle déclenché par une résonance trop intense entre ce qu'il tente d'ignorer de lui-même et ce qu'il vit.

\section{Effet de clivage}

Le clivage, mécanisme de défense qui participe de la construction de l'appareil psychique, est en lui-même un paradoxe, il maintient ensemble en les isolant deux impossibles, deux termes contradictoires. "Les deux réactions au conflit, réactions opposées, se maintiennent comme noyau d'un clivage du moi" (Freud, 1938, p. 284). Ainsi le clivage comme mécanisme de défense ne règle rien, il supprime la question; cette suppression, cette mise hors-Psyché, pour être maintenue, demande de l'énergie sous peine de se faire, à nouveau, entendre. Le clivé, aussi, peut faire retour, selon l'expression de René Roussillon. L'effet de paradoxe dont il est question à propos du suicide est celui d'un clivage fragilisé au point de laisser surgir les inconciliables. Ce qui était séparé se mélange, les espaces se confondent et leur contenu également. La situation est souvent dite aconflictuelle, elle est, à mon sens, non conflictualisée et ne peut l'être suite à la confusion interne que l'émergence d'une expérience non traitée, vécue et non 


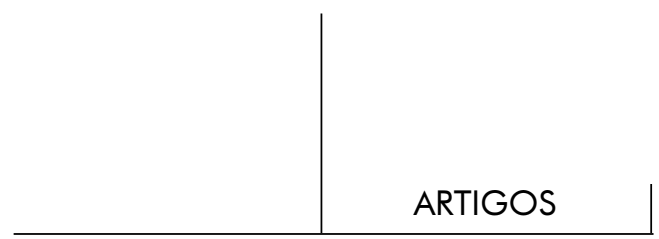

éprouvée (Winnicott, 1974) produit. Le conflit oppose deux énoncés issus de lieux différenciés alors que la crise psychique est un moment d'indifférenciation, un bouleversement de l'appareil psychique. Celui-ci est atteint dans ses fondations et envahi par des éléments d'une inquiétante étrangeté réduisant le potentiel conflictuel. L'inquiétant (Freud, 1919) est défini par cette difficulté particulière d'en situer l'origine: dedans/dehors, ancien/nouveau, connu/inconnu, illusion/ perception, moi/non-moi,...

Chantal, suite au décès de son père, a traversé un épisode dépressif. Au moment de son geste, elle "paraissait en être sortie", au moins aux yeux de son entourage. Au-delà de la perte dans la réalité restait la perte inconsciente, ignorée, celle que Chantal veut dépasser pour vivre sa vie, celle qui oblige à un renoncement de nature essentiellement narcissique et qui atteint les fondations en nécessitant au minimum un pas de côté, au maximum un grand écart. Chantal, proche de son père, prend en contre point ce que lui semble être la relation entre ses deux parents, une relation tyrannique et cause des souffrances du père. La qualité particulière de cette relation, celle de maintenir lié, est au minimum remise en question, au maximum annulée par le suicide du père. La phrase répétée par Chantal, cette bouée percée, tente de traiter la question du meurtre: si on ne peut pas l'empêcher alors elle ne peut en être coupable. Ce qui s'entend, sans un mot, c'est la tromperie (si ce n'est pas elle qui l'a tuée alors c'est lui qui l'a laissée tomber) dont la relation au mari fera les frais. Son pacte de mariage, la menace de suicide, devient caduc, à cette place elle ne peut plus "se supporter". Être meurtrière ou être annulée, de quoi est-il le plus vital de se défendre? Se séparer de son époux réactive une expérience qu'en son temps Chantal n'a pu traiter et à laquelle, suite au décès du père, elle est à nouveau confrontée comme pour la première fois. Le projet de séparation s'inclut dans le travail d'élaboration, de mise en sens d'un vécu en manque d'inscription. Celui-ci peut être situé du côté de la relation de Chantal au couple parental: la préférée du père auprès duquel elle se définissait à l'opposé de sa propre mère. "Je veux vivre ma vie" semble une acquisition nouvelle, le fruit de la reprise du travail complexe de la destructivité qui, de l'attaque meurtrière des premiers objets à l'expérience de leur survie, ouvre sur un ailleurs, sur d'autres objets.

\section{Perte et répétition}

La clinique confirme que la problématique de la perte est toujours présente chez le sujet "suicidaire". La réaction à la perte, le mode de traitement psychique de celle-ci par le sujet, révèle la qualité de l'investissement de l'objet perdu. La 
perte n'est pas uniquement celle de l'objet réel mais aussi et, peut-être même surtout, celle de la relation à cet objet. Dans le cas de la déception, l'objet réel n'est pas simplement perdu, il n'a pas répondu aux attentes du sujet; c'est la relation à l'objet (idéal) qui menace de l'être.

La perte n'est pas souvent évoquée comme telle, il est question de décès, de divorce, d'adultère, ou d'une succession d'évènements de cet ordre. Ce n'est pas l'événement lui-même, si dramatique soit-il, qui amène le sujet à attenter à sa vie. L'événement ou les événements évoqués, sans être historiques, ont eu lieu dans les années précédentes. Les liens entre événements douloureux et tentative de suicide ne sont donc pas immédiatement reconnus. Ce n'est pas l'événement douloureux qui est en cause mais les remaniements psychiques qu'il provoque chez le sujet, le "travail de deuil" dit-on communément dans le cas du décès d'un proche.

Entre le deuil et la mélancolie, il y a de nombreuses variations d'éprouvés dépressifs dont les mécanismes peuvent être différenciés. Côté deuil, un travail de désinvestissement et réinvestissement, côté mélancolie, un mouvement d'identification narcissique et une faillite de l'investissement libidinal, objectal.

Si le mélancolique se défend à la fois de l'objet devenu mauvais et de l'obligation de l'abandonner, c'est qu'en abandonnant sa relation à l'objet, il s'abandonne lui-même. Ainsi, l'objet, dans sa réalité, est aussi porteur de cette différenciation moi/non-moi, y renoncer installe un état d'indifférenciation, quand "avoir" et "être" se confondent.

Le sujet qui commet un geste suicidaire de nature impulsive est confronté à un objet dont il ne peut se défendre en s'éloignant. La capacité du moi à lier, mise en échec dans l'état de crise, passe par l'objet. Son manque, par absence dans la réalité (dans la situation originaire), ou par impossibilité pour le sujet d'y avoir recours (la relation du sujet envers l'objet ne permet pas cette liaison), signe la fragilité de sa constitution et renvoie le sujet à sa propre fragilité: le sentiment d'être vacille. Le moment suicidaire est en lien avec un état de tension psychique intense lié à la réactualisation soudaine d'une expérience de renoncement impossible. L'objet vécu comme détruit, ce que décrit André Green avec le complexe de la "mère morte", ne peut être intériorisé, un trou se crée dans l'appareil psychique, un espace de non-liaison que le sujet doit colmater sous peine de se sentir défait. De "n'avoir", on ne peut "être". De même, un objet trop fragile qui, face à la destructivité du sujet, menace d'être détruit ne permet pas le traitement de l'ambivalence. Le sujet ne peut perdre l'objet, il ne peut l'avoir, il peut le devenir en conjuguant différemment "avoir" et "être", en les confondant.

L'une des issues psychiques, celle qui me paraît essentiellement en œuvre dans la clinique qui m'occupe, est l'identification défensive. L'objet est rendu inattaquable dans la confusion sujet-objet. Par ce processus le sujet s'approprie

Rev. Latinoam. Psicopat. Fund., São Paulo, v. 15, n. 4, p. 766-780 dezembro 2012 


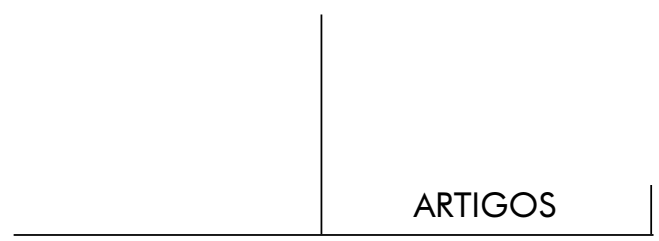

l'objet en le prenant à revers. Ceci à lieu au prix d'une part de lui-même devenue silencieuse, sans adresse de n'avoir pu être psychiquement traitée. Cette connaissance de soi, à la fois perdue et enkystée, peut faire retour. Sous une forme très légère, ce retour équivaut au sentiment de l'inquiétante étrangeté, sous une forme beaucoup plus intense, il est traumatique. Dans tous les cas, l'effet est dû à la venue à la conscience d'un intime étranger, d'un étranger d'être trop intime. Cet accès à la conscience est double d'une certaine façon car, c'est d'être "appelé" de l'extérieur qu'il trouve la voie de l'intérieur. Ce sont les deux coups du trauma, deux coups non simultanés qui ne se révèlent que simultanément, que réciproquement, l'un donnant toute sa force à l'autre jusque-là ignoré. Le trauma est une ouverture psychique, une effraction qui nécessite un travail de liaison, d'élaboration de ce qui là se dévoile. Le geste suicidaire tente, en répétant, une élaboration de l'insensé.

L'énoncé répété par Chantal, "quand quelqu'un veut vraiment mourir, on ne peut pas l'en empêcher", est une tentative d'arrimage alors que les failles menacent de devenir des gouffres sous l'effet de la réactualisation d'événements psychiques restés, jusque-là, suffisamment silencieux. Le tremblement n'est pas tant le décès du père, que ce que le traitement psychique de cette perte révèle du côté de la relation à l'objet maternel; le contre-investissement manifeste de la relation à sa mère n'a d'équivalent que l'investissement de la relation à son père et par extension l'identification à l'objet maternel. À cette place, plus confuse que définie, elle ne se connaît plus quand celle-ci lui est révélée. Dans le cas de Chantal, le geste suicidaire est à la fois un retour vers la situation qui précède le coup, la relation apaisante à son père, et une ouverture vers un ailleurs; la relation à laquelle elle met fin, n'est-ce pas celle qu'elle a déjà perdue? Le suicide n'est plus cette menace qui liait Chantal à ses premiers objets et à partir de laquelle un pacte inconscient fondait son mariage. Son geste suicidaire ne tente-t-il pas le traitement de fantasmes inconscients, que la menace n'a pas permis de traiter à l'origine, et que la séparation à venir réactive, réactualise? Psyché, théâtre de la vie fantasmatique, est soudainement envahie par un afflux d'excitation quand fantasme et réalité se font écho, quand deux scènes n'en font qu'une.

\section{La scène suicidaire ou le théâtre en feu}

Le geste suicidaire a lieu alors que le monde interne est le théâtre d'une scène désordonnée dont le scénario met "le feu au théâtre". Une scène originaire qui tente "de donner figure et lumière à ce qui doit sa violence à l'ombre de l'informe" (La scène primitive et quelques autres, p. 4). Scène cachée, elle a lieu dans l'espace 
de la skenè du théâtre antique (les coulisses), elle nourrit ce qui a lieu dans l'espace nommé proscenium, l'espace situé devant. La skenè est le théâtre invisible, théâtre de l'invisible, de l'indifférencié, de l'informe: les acteurs changent "de masques, de rôles donc d'identité ou de sexe" (Ibid).

Entre proscenium et skenè, le rideau perd de son opacité, les deux scènes se mélangent, se confondent et le théâtre s'embrase. La représentation, au sens de la pièce qui se joue ici et maintenant, doit trouver un autre théâtre sous peine d'effondrement de l'édifice culturel.

Entre les deux scènes, les liens ne peuvent se représenter. Ils existent, mais perdent leur qualité, celle de maintenir ensemble, de synthétiser, dès qu'ils sont mis à nu. Skenè, est une scène à l'origine d'une autre scène qu'elle organise, skenè doit rester cachée. D'un théâtre à l'autre, de psychè à soma, tel serait le déplacement, de la scène interne, inscrit dans le geste suicidaire. Cette scène aurait les qualités des Urszenen dans sa première définition par Freud: expériences infantiles traumatisantes organisées en scénario. Scène du traumatisme originaire avec laquelle le sujet a construit l'autre scène de son théâtre interne, proscenium, scène de la représentation tournée vers l'extérieur et habillée de l'intérieur. Construit avec et non malgré, car, comme au théâtre, sans coulisses pas de représentation. Le geste suicidaire constitue un changement de théâtre, de Psyché à Psyché-Soma, le retrait du spectateur-acteur d'une scène dans laquelle il ne peut plus être.

Le sentiment "d'être au bord du gouffre", à la limite "d'être", serait conséquent à l'effondrement d'un clivage profond. Ce sentiment résulterait essentiellement d'une perte d'opacité des limites de l'espace interne qui laisserait éprouver l'expérience d'un non-moi au sein du moi. Cette "élongation" met en danger le moi par un excès de représentations débordant ses capacités à lier, à contenir l'ensemble, afin qu'une pièce puisse se jouer; une pièce, une histoire dans le temps et dans l'espace, tissée selon les règles des processus secondaires. Le geste suicidaire serait le recours ultime du moi pour "mettre fin" à une scène où se joue sa propre disparition. Le fort sentiment de "renaître" qu' expriment certains patients après un geste suicidaire est peut-être à entendre en lien avec cet éprouvé.

La scène suicidaire décrite par Chantal renvoie à une scène historique, familiale, où tyrannie et menace de la mort font écho à l'impossible séparation. Elle s'en extrait en la rejouant, en la menant à son terme, pourrait-on dire. Cette scène, cet acte suicidaire parle d'elle, de son histoire rejouée là, à son insu. Elle est actrice, spectatrice et metteur en scène, dans l'actuel, d'un scénario d'un autre temps, premier temps, temps originaire. 


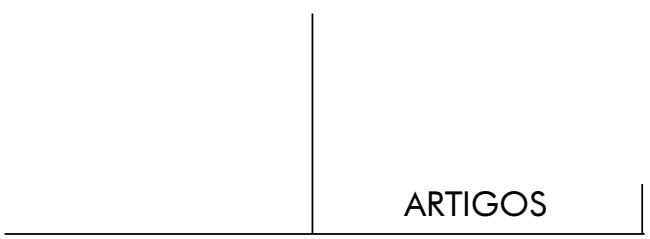

Après-coup

Que dire après ce parcours de ce "nouvel élan" qui suit parfois un geste suicidaire?

Par son geste, Chantal a évité la séparation tout en la concrétisant, elle n'est plus retournée dans la maison conjugale. Physiquement, elle a survécu à son geste, psychiquement elle a survécu à la séparation. Plus qu'une survie, c'est d'une "vie" dont elle dit bénéficier, comme si la "survie" c'était avant. Le geste suicidaire peut être un début... quand le sujet en réchappe. Il signe la nécessité d'une fin, d'un achèvement qui ne peut être pensé, seulement agi. Il est également une répétition, la pièce est rejouée. Elle peut n'être que "le retour éternel du même" et ouvrir sur un scénario différent ou relever de "la répétition indéfinie de l'identique" (De M'Uzan, 1969, p. 84) et rester fidèle au dénouement du premier texte.

Le geste suicidaire cherche une "signification" à ce qui a toujours été un "insensé". Il constitue une mise en forme, avant une possible mise en sens, d'une expérience non élaborée et maintenue, sous l'effet d'un clivage, exclue à l'intérieur même de Psyché. Cette mise en forme secondaire d'une expérience ancienne est un effet d'après-coup: une scène actuelle donne son sens à une scène restée insensée car vécue à une époque où le sujet n'avait pas les moyens de sa représentation. La tentative de suicide comme tentative d'après-coup psychique serait une réponse à "ce nouvel élan" qui signerait une réussite, une ouverture psychique, une re-liaison là où un clivage maintenait les énergies déliées. Dans la clinique des sujets suicidaires, et dans le cas de Chantal, peut-on penser que la destruction du clivage fait suite à sa fragilisation et qu'un mécanisme de défense plus souple, tel le refoulement, peut-être accessible au sujet? Les cas d'addiction au suicide, décrit dans la littérature, renverraient à l'image d'un clivage "indestructible" contre lequel le sujet viendrait buter tentant, par la répétition, un accès à la représentation d'une expérience dont il n'est pas sorti "vivant" mais dans la répétition de laquelle il n'est pas encore un "survivant".

La scène suicidaire exprime quelque chose de la scène interne, des scénario fantasmatiques qui s'y jouent, mais de façon énigmatique comme sait le faire l'inconscient. Elle condense en elle d'autres scènes, ceci se repère cliniquement quand, à la suite de sa remémoration, une ouverture psychique se dévoile qui peut permettre qu'un récit différent advienne. Cette condensation particulière, autour de la scène suicidaire, évoque la condensation signifiante de la scène du rêve. C'est sur le modèle de l'après-coup que l'histoire de la scène peut s'inscrire comme histoire, si un autre en est le témoin. C'est cette historisation potentielle qui me paraît être une voie d'accès intéressante pour poursuivre la réflexion sur l'énigme du suicide, ou de sa tentative. 


\section{Références}

Conférence de consensus (2001). La crise suicidaire: reconnaître et prendre en charge. Paris: J Libbey Eurotext/Fédération Française de Psychiatrie. (Trabalho original publicado em 2000).

De M'Uzan, M. (1977). Le même et l'identique. In De l'art à la mort (pp. 83-97). Paris: Gallimard. (Trabalho original publicado em 1969).

Freud, S. (1988). Deuil et mélancolie. In Oeuvres Complètes (Vol. XIII, p. 261-278). Paris: PUF. (Trabalho original publicado em 1915).

Freud, S. (1996). L’inquiétant. In Oeuvres Complètes (Vol. XV, pp. 147-188). Paris: PUF. (Trabalho original publicado em 1919)

Freud, S. (1974). Psychogenèse d'un cas d'homosexualité féminine. In Névrose, psychose et perversion (pp. 245-270). Paris: PUF. (Trabalho original publicado em 1920)

Freud, S. (1985). Le clivage du moi dans le processus de défense. In Résultats, Idées, Problèmes - Tome II, 1921-1938 (pp. 283-286). Paris: PUF. (Trabalho original publicado em 1938)

Green, A. (1982). La mère morte. In Narcissisme de vie, narcissisme de mort (pp. 222254). Paris: Édition de Minuit. (Trabalho original publicado em 1980)

Kaës, R. et al., (1990). Crise, rupture et dépassement: analyse transactionnelle en psychanalyse individuelle et groupale. Paris: Dunod. (Trabalho original publicado em 1979)

La scène primitive et quelques autres (1992). Nouvelle Revue de Psychanalyse, 46.

Les premiers psychanalystes. Minutes de la société psychanalytique de Vienne (1978). Tome II, 1908-1910. Paris: Gallimard.

McDougall, J. (1978). Narcisse en quête d'une source. In Plaidoyer pour une certaine anormalité (pp. 139-160). Paris: Gallimard. (Trabalho original publicado em 1976)

Roussillon, R. (2004). Agonie, clivage et symbolisation. Paris: PUF. (Trabalho original publicado em 1999)

Winnicott, D. W. (1975). La crainte de l'effondrement in Figures du vide. Nouvelle Revue de Psychanalyse, 11, 35-44. 


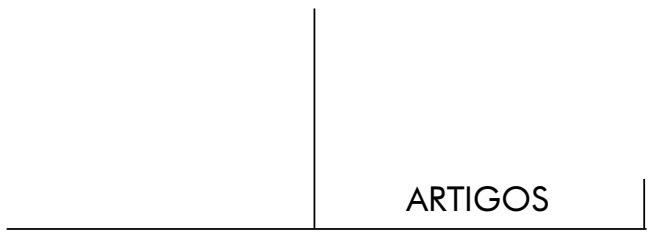

Resumos

(Do sem sentido do gesto suicida à condensação significativa do suicídio)

O gesto suicida é um enigma, "como o ego pode consentir a sua própria autodestruição" questiona-se Freud en 1915. Este artigo, partindo desta constatação teórica e clínica e apoiado na história de Chantal, propõe a seguinte via de reflexão: o gesto suicida inclui uma primeira cena que constitui uma primeira repetição ou é o segundo tempo do trauma. Esta reatualização traumática pode ser seguida de um movimento de retomada da história do sujeito a partir do momento insensato originário do gesto.

Palavras-chave: Gesto suicida, paradoxo, a posteriori (après-coup), cena suicida suicide)

(From the senselessness of suicidal gestures to the signifying condensation of

An act of suicide is an enigma. "How can the ego consent to self-destruction?" asked Freud in 1915. Based on this theoretical and clinical observation and on the story of Chantal, this article presents the following approach: either a suicidal act includes a first scene that constitutes a first repetition, or it is the second moment of a trauma. This traumatic repetition may be followed by a re-enactment of the subject's story as of the original and senseless moment of the gesture.

Key words: Suicidal gesture, paradox, après-coup, suicide scene

(Del sin-sentido del gesto suicida a la condensación significativa del suicidio)

El acto suicida es un enigma, “ ¿cómo el ego puede dar su consentimiento a su autodestrucción?”, se pregunta Freud en 1915. Con base en esta constatación teórica y clínica, el presente artículo, basado en la historia de Chantal, se propone a pensarla como una forma de "après-coup". El acto suicida incluye una escena que es una primera repetición, o el segundo tiempo del trauma. Esta re-actualización traumática puede ser seguida por un movimiento de retomada de la historia del sujeto a partir del momento originario e insensato del gesto.

Palabras claves: Gesto suicida, paradoja, "après-coup”, escena suicida

(Von der Sinnlosigkeit der selbstmörderischen Handlung bis zur bedeutungsvollen Kondensation des Selbstmordes)

Die selbstmörderische Handlung ist ein Rätsel. Freud fragt sich 1915: „Wie kann das Ego der Selbstzerstörung zustimmen?". Ausgehend von der theoretischen und klinischen Beobachtung und aufgrund der Geschichte von Chantal wird in diesem Beitrag folgende Reflexion angestellt: Die selbstmörderische Handlung beinhaltet eine erste Szene, die eine erste Wiederholung darstellt, $d$. h. es ist ein zweiter Teil des 
Traumas. Auf diese traumatische Wiederholung kann eine Bewegung des unsinnigen Auslösermoments folgen.

Schlüsselwörter: Selbstmörderische Handlung, Paradox, das a posteriori, selbstmörderisches Szenarium

Citação/Citation: André, J. \& Chouvier, B. (2012, dezembro). De l'insensé du geste suicidaire à la condensation signifiante de la scène suicidaire. Revista Latinoamericana de Psicopatologia Fundamental, 15(3), 766-780.

Editor do artigo/Editor: Prof. Dr. Manoel Tosta Berlinck

Recebido/Received: 27.10.2011 / 10.27.2011 Aceito/Accepted: 15.2.2012 / 2.15.2012

Copyright: (C) 2009 Associação Universitária de Pesquisa em Psicopatologia Fundamental/ University Association for Research in Fundamental Psychopathology. Este é um artigo de livre acesso, que permite uso irrestrito, distribuição e reprodução em qualquer meio, desde que o autor e a fonte sejam citados / This is an open-access article, which permits unrestricted use, distribution, and reproduction in any medium, provided the original author and source are credited.

Financiamento/Funding: Os autores declaram não ter sido financiados ou apoiados / The authors have no support or funding to report.

Conflito de interesses/Conflict of interest: Os autores declaram que não há conflito de interesses / The authors declare that has no conflict of interest.

\section{JoAnNe ANDRÉ}

Psychologue clinicienne, Doctorante et chargée de cours, rattachée au Centre de Recherche en Psychologie et Psychopathologie Clinique (CRPPC), Université Lyon 2, Institut de Psychologie.

56, rue Nationale

38370 Les Roches de Condrieu

Fone: $(00+21)+33474565241 /+33675368788$

e-mail: joanne.andre@univ-lyon2.fr

\section{Bernard Chouvier}

Professeur de psychopathologie, Centre de recherche en psychopathologie et psychologie clinique (CRPPC), Université Lyon 2, Institut de Psychologie.

5, Avenue Pierre Méndes

France 69676 Bron Cedex

Rev. Latinoam. Psicopat. Fund., São Paulo, v. 15, n. 4, p. 766-780 dezembro 2012 